\title{
La comunicación hospitalaria 2.0: un nuevo desafío para la comunicación institucional
}

\section{Pablo Medina Aguerrebere \\ Facultat de Ciències de la Comunicació de la Universitat Internacional de Catalunya}

Recibido: 26 de mayo de 2012

Aceptado: 5 de septiembre de 2012

\section{Resumen}

En estos últimos años, el contexto hospitalario ha estado sometido a varios cambios, entre los que destacan los provocados por el interés creciente que la gente manifiesta hacia los temas sanitarios, el desarrollo tecnológico y la implementación de internet, especialmente las aplicaciones 2.0 (blogs, redes sociales, etc.). Esta situación supone un reto para las instituciones hospitalarias, las cuales se caracterizan por la escasa tradición que tienen en la gestión profesional de la comunicación institucional. Las distintas aplicaciones de internet 2.0 constituyen una oportunidad para que los hospitales implanten estrategias de comunicación institucional que les permitan mejorar sus relaciones comunicativas con los diferentes stakeholders. El objetivo de este artículo de revisión bibliográfica es comprender cuáles son los criterios principales que debe considerar un hospital a la hora de definir su estrategia de comunicación institucional 2.0. La conclusión del artículo indica que dichos criterios son cinco: 1) combinar el enfoque comercial con el enfoque pedagógico centrado en la formación del paciente en temas sanitarios; 2) implicar a los profesionales de la salud; 3 ) complementar las acciones de comunicación 2.0 con acciones de comunicación interpersonal que tengan lugar en el hospital de modo presencial; 4) adecuar la estrategia de comunicación 2.0 a la política de comunicación institucional del hospital y 5) respetar la ética comunicativa y hospitalaria.

Palabras clave: hospital; comunicación institucional; comunicación en línea; web 2.0.

\section{Hospital communications 2.0: a new challenge for institutional communication}

\section{Abstract}

In recent years, the hospital context has been subjected to several changes, among them, those caused by the increased interest people have shown towards health issues, technological development, and the implementation of the Internet, especially Web 2.0 tools (blogs, social media, etc.). This situation presents a challenge for hospitals, characterized by a limited tradition in professional management of institutional communications. Web 2.0 tools offer 
an opportunity for hospitals to implement institutional communication strategies that might enable them to improve their communicative relationships with different stakeholders. The objective of this paper, which reviews the available literature, is to understand the main criteria that must be considered by a hospital in order to determine its strategy of institutional communications 2.0. The conclusions arrived at by this paper indicate that these criteria should be five: 1) combine the commercial approach with a pedagogical approach focused on educating the patient on health issues; 2 ) involve health professionals; 3) complement communications 2.0 with interpersonal face-to-face communications held within the hospital; 4) adapt communications 2.0 strategies to the hospital's institutional communication norms; and 5) respect communication and hospital ethics.

Keywords: hospital; institutional communication; online communication; web 2.0.

\section{A comunicação da administração hospitalar 2.0: um novo desafio para a comunicação institucional}

\section{Resumo}

Em anos recentes,o contexto da administraçao hospitalar tem passado por diversas mudanças, especialmente pela ênfase causada pelo interesse crescente em assuntos sanitários, o desenvolvimento tecnológico e o surgimento da internet, especialmente aplicações 2.0 (blogs, redes sociais, etc.). Esta situação supõe um desafio para as instituições hospitalares, que são caracterizadas pela pouca tradição que têm na gerência profissional da comunicação institucional. As diferentes aplicações da internet 2.0 constituem uma oportunidade de modo que os hospitais implantem as estratégias de comunicação institucional que permitem melhorias nas suas relações comunicativas com diferentes stakeholders. O objetivo deste artigo de revisao bibliográfica é compreender quais são os critérios principais que um hospital considera na hora de preparar sua estratégia de comunicação institucional 2.0. A conclusão do artigo indica que estes critérios são cinco: 1) combinar a aproximação comercial com a abordagem pedagógica centrada na formação do paciente em assuntos sanitários; 2) envolver os profissionais da saúde; 3) complementar as ações de uma comunicação $2.0 \mathrm{com}$ as ações de uma comunicação interperssoal que ocorrem no hospital da maneira real; 4) adaptar a estratégia de uma comunicação 2.0 à política de uma comunicação institucional do hospital; e 5) respeitar as éticas comunicativa e hospitalar.

Palavras chave: hospital; comunicação institucional; comunicação online; web 2.0.

\section{Introducción}

Las organizaciones hospitalarias tienen, en su mayoría, un carácter público, es decir, no poseen ánimo de lucro, lo cual hace que la concreción de ciertas acciones de comunicación comercial sea más difícil que en otro tipo de organizaciones empresariales privadas. A pesar de esta realidad, cada vez más organizaciones hospitalarias apuestan por la implementación de una gestión profesional de la comunicación institucional. Además, en estos últimos años, el interés creciente que la 
gente manifiesta hacia los temas relativos a la salud, la implementación de las nuevas tecnologías de la comunicación y de la información (TIC) y la adopción por parte del ciudadano de una actitud cada vez más participativa y activa, son algunos de los factores que han cambiado de modo radical las estrategias de comunicación institucional desarrolladas por los hospitales. En este contexto, el desarrollo reciente de la denominada medicina 2.0 (blogs, redes sociales, telemedicina, etc.) supone un nuevo reto comunicativo para las instituciones hospitalarias, las cuales son conscientes de las implicancias de estas aplicaciones para la salud pública. Sin embargo, desde un punto de vista institucional, ¿cuáles son los criterios principales que debe considerar un hospital a la hora de fijar su estrategia de comunicación institucional 2.0? Para intentar responder a esta pregunta el artículo analiza, en primer lugar, el contexto hospitalario; en segundo lugar, la comunicación institucional en los hospitales; en tercer lugar, la comunicación hospitalaria en línea; y en cuarto término, antes de las conclusiones, la comunicación hospitalaria 2.0.

\section{Contexto hospitalario}

Según la Real Academia Española (2001), se define al hospital como el establecimiento destinado al diagnóstico y tratamiento de enfermos, así como a la investigación y a la enseñanza. El aspecto más relevante de esta definición es que identifica las tres dimensiones del hospital: asistencia a pacientes, investigación clínica y enseñanza universitaria. Basándonos en Asenjo (2002), los hospitales pueden clasificarse según cinco criterios: a) función profesional (hospital general u hospital especializado), b) tipo de pacientes (hospital de corta, larga o media estancia), c) ámbito demográfico (hospital regional, nacional o internacional), d) nivel asistencial (hospital de baja complejidad, media complejidad o tecnología de punta) y e) dependencia patrimonial (hospital público o privado). Independientemente del tipo de hospital del que se trate, todos ellos asumen unas funciones específicas, entre las que destacan el cuidado del paciente, el fomento de la enseñanza, la promoción de la investigación y el apoyo a otros centros hospitalarios (McKee, Healy, 2002).

En este contexto organizacional en el que los hospitales asumen tantas funciones, resulta fundamental la existencia de profesionales del management de la gestión que coordinen las diferentes actividades emprendidas por el hospital. El gestor profesional, según Mintzberg, "se reivindica como una persona que trabaja en proyectos múltiples, involucrada en la puesta en marcha de técnicas neutra- 
les para cualquier necesidad de gestión" (Mintzberg, 2004: 153). Y para resolver dichas necesidades, una de las herramientas clave de las que dispone el gestor es la comunicación. De hecho, según Mucchielli, la gestión es "el arte de resolver, a través de distintas formas de comunicación interna, los problemas organizacionales comunes y recurrentes que se presentan a todo responsable" (Muchielli, 2005: 184). En este contexto, cada vez más hospitales han decidido apostar por la realización de actividades de comunicación, lo cual ha favorecido el desarrollo de la comunicación institucional hospitalaria.

\section{Comunicación institucional en hospitales}

En estos últimos años, cada vez más hospitales han decidido implantar un departamento de comunicación institucional. Una de las responsabilidades más importantes que asume ese departamento es la definición de los elementos constituyentes de la política de comunicación institucional del hospital, es decir la identidad, la misión, la visión, la cultura y la imagen. La fijación de estos elementos ayuda al hospital a establecer su posicionamiento estratégico, lo cual es clave en un contexto caracterizado por el aumento de la competencia, el desarrollo de los grupos hospitalarios privados, la creación de hospitales presentes en diferentes países y el interés creciente que la población manifiesta hacia los temas sanitarios, lo cual implica una exposición social cada vez mayor por parte de los hospitales.

En lo que se refiere a la identidad de una institución, Costa la define como "un sistema de comunicación que se incorpora a la estrategia global de la empresa y se extiende y está presente en todas sus manifestaciones, producciones, propiedades y actuaciones" (Costa, 2001: 218). Una vez que el hospital ha fijado su identidad es necesario comunicarla a nivel externo, ya que gracias a esto, según Van Riel y Fombrun (2007), se crea una cohesión interna entre los empleados y la organización, así como entre la organización y sus stakeholders. Junto con la identidad, el hospital establece de un modo preciso cuál es su misión. Las organizaciones se crean para alcanzar una meta, unos objetivos que son su misión en la sociedad, los cuales pueden ser exclusivamente económicos, sociales o comunitarios (Nieto, 2008). La misión es un elemento clave en el posicionamiento estratégico del hospital ya que, según Parsons (2001), ayuda a la institución a conocer mejor a su público, a establecer las prioridades estratégicas y a definir el tono de la comunicación institucional.

Además de la identidad y de la misión, el hospital fija los otros tres elementos constituyentes de su política de comunicación institucional: la visión, la cultura y 
la imagen. Barret considera que "la visión representa un nivel de motivación más profundo que una misión. La misión describe los medios, la visión describe el objetivo y establece una declaración cautivadora de lo que la organización intenta conseguir" (Barret, 2003: 83). En lo que concierne a la cultura institucional, se trata del conjunto de creencias y prácticas de los empleados del hospital, es decir, hace referencia al modo de trabajar y de comportarse de los profesionales que desempeñan su labor en la organización hospitalaria. Y para la creación y promoción de dicha cultura, la comunicación juega un rol esencial (Parsons, 2001; Wright, Sparks, O’Hair, 2008). Por último, la imagen del hospital es un elemento que es gestionado por el propio hospital, pero que está muy influenciado por sus grupos de interés (stakeholders). Van Riel afirma: "las impresiones personales, la comunicación interpersonal y la comunicación de los medios masivos de comunicación se combinan para producir una mezcla de impresiones reales y paralelas cuya totalidad forma la imagen" (Van Riel, 1998: 95).

La definición de los cinco componentes que conforman la política de comunicación institucional (identidad, misión, visión, cultura e imagen) resulta esencial para la correcta inserción de la comunicación institucional en los hospitales. Y para ello, el departamento de comunicación necesita contar con la colaboración de la dirección del hospital. Según Argenti, "los directivos necesitan ser completamente conscientes del impacto tremendo de la identidad, de la imagen y de la reputación y deben aprender a gestionar estos recursos" (Argenti, 2003: 76).

\section{Comunicación hospitalaria en línea}

En este contexto en el que los numerosos avances que tienen lugar en la medicina la convierten en una ciencia aún más compleja, hay un aumento de la información sanitaria disponible en internet (Reid, 2008). La red ha supuesto una verdadera revolución en el ámbito de la comunicación institucional hospitalaria; sin embargo, también plantea una serie de retos que deben considerarse.

En primer lugar, los sistemas de información de los hospitales deben proteger la vida privada de los pacientes (Rafalski, Mullner, 2003), lo que se plasma en que las oportunidades de comunicación ofrecidas por internet deben ser coherentes con el respeto de las diferentes leyes sobre privacidad y confidencialidad que se aplican en los hospitales.

En segundo lugar, internet se ha convertido en una verdadera amenaza que puede afectar a la confianza que el paciente tiene en la relación terapéutica esta- 
blecida con el profesional de la salud (Fostier, 2005), lo cual supone un riesgo de salud pública en el sentido de que, a pesar de todas las informaciones médicas que circulan por la red, el profesional de la salud que trabaja en un centro hospitalario es $-\mathrm{y}$ siempre seguirá siendo- la mejor fuente de información médica de la que dispone un paciente.

En tercer lugar, la ética debe estar presente en todas las acciones de comunicación y salud (Guttman, 2003), lo cual se traduce en la consideración de internet como una herramienta cuyos objetivos prioritarios son ayudar al profesional de la salud a hacer mejor su trabajo, por un lado, y a facilitar la recuperación terapéutica del paciente, por otro.

En estos últimos años, cada vez más hospitales de todo el mundo han apostado por la creación de un sitio web para mejorar así las relaciones comunicativas que mantienen con sus diferentes stakeholders, como por ejemplo los pacientes, los familiares de pacientes, las autoridades sanitarias o los medios de comunicación. El desarrollo de dicha página web es asumido por el departamento de comunicación del hospital, el cual, en colaboración con la dirección de la institución hospitalaria, los profesionales de la salud que trabajan en dicho centro e, incluso en ciertas ocasiones, los organismos externos (agencias de publicidad, diseño, etc.), define una estrategia de comunicación en línea que satisfaga las necesidades del hospital y de sus grupos de interés. Para la realización de ese sitio los hospitales consideran cinco criterios principales: a) enfoque pedagógico, b) accesibilidad, c) personalización, d) interactividad y e) promoción de la comunicación interpersonal.

El enfoque pedagógico subraya la importancia de internet como herramienta que favorece el aprendizaje. El poder de la red como herramienta pedagógica (Sierra Caballero, 2002) ha hecho que varias instituciones sanitarias utilicen esa herramienta para transmitir a los pacientes diferentes informaciones sobre los hábitos de vida saludable, como por ejemplo los relativos a las alergias, la obesidad y el cáncer (Moyá Garrido, 2009).

En lo que concierne a la accesibilidad, los hospitales priorizan los diseños que facilitan el uso del sitio web ya que, según Fisher et al. (2008), las páginas especializadas en salud que son consideradas como efectivas transmiten una imagen de calidad fiable, de confianza, de inteligencia y sobre todo, de simplicidad en el uso. Este tipo de páginas web evolucionan de la difusión de informaciones generales a la satisfacción de las necesidades informativas precisas de los pacientes (Huntington, Nicholas, Williams, 2003). 
Con respecto del criterio de personalización, según Giustini (2006), la búsqueda de informaciones médicas personalizadas es una realidad cada vez más importante, ya que el caso de cada paciente siempre es distinto. Además, el uso de internet para conseguir informaciones médicas está muy unido a la intimidad de las experiencias sanitarias vividas por cada paciente (Kivits, 2009).

En lo que concierne al cuarto criterio, la interactividad, los pacientes, según Buckley (2007), cada vez dan más importancia a la búsqueda de experiencias médicas que a la búsqueda de informaciones. Esta realidad ha llevado a varios hospitales a apostar por la denominada comunicación en línea 2.0, la cual se plasma en diversas aplicaciones como los blogs o las redes sociales.

Por último, el quinto criterio se centra en la promoción de la comunicación interpersonal entre el paciente y el profesional de la salud. Es decir, el sitio en internet no puede aislar al paciente del resto de las fuentes de información que utiliza, como por ejemplo el profesional sanitario (Lemire, 2009). Por ello, es necesario que en dicha página el paciente disponga de diferentes herramientas para contactar con el profesional que lo atendió, como por ejemplo el número de teléfono, el correo electrónico, etc.

\section{Comunicación hospitalaria 2.0}

La incorporación de las TIC en el mundo sanitario se ha producido en dos oleadas: la primera tuvo como eje los programas de gestión, como el CRM (customer relationship management) o el SCM (supply chain management); y la segunda, se centró en las tecnologías 2.0, las cuales fomentan la participación entre pacientes y profesionales de la salud (Chui, Miller, Roberts, 2009). La tecnología 2.0 ha supuesto un cambio importante en las estrategias de comunicación institucional de los hospitales. Dicha tecnología ha facilitado que el paciente se sitúe en el centro de todas las iniciativas de comunicación emprendidas por el hospital, lo cual supone una oportunidad, pero también un riesgo. Es una oportunidad, ya que la evolución de la web estática (web 1.0) a la web participativa (web 2.0) permite eliminar barreras espaciotemporales y fomentar la comunicación activa entre el hospital y sus diferentes stakeholders. Pero también supone un riesgo ya que permitir a todo tipo de pacientes la creación, la consulta y la difusión de contenidos médicos constituye una acción arriesgada, especialmente cuando dichos pacientes no poseen un nivel elevado de conocimiento sobre dichos temas. Por otro lado, según Hesse et al. (2005), dentro de poco los médicos serán considerados como meros colaboradores 
que ayudan al paciente a acceder a diferentes datos sanitarios basados en una gran de cadena de información personalizada y mediatizada. Por ello, la estrategia de comunicación institucional del hospital debe hacer frente a esta tendencia y resaltar el valor del profesional de la salud como fuente de información médica y como protagonista principal - junto con el paciente- de la comunicación hospitalaria 2.0. Para ello, el hospital puede recurrir a varias iniciativas, entre las que se destacan tres: a) los blogs, b) las redes sociales y c) las aplicaciones del teléfono móvil.

En el año 2008, el número de blogs escritos en inglés y especializados en temas médicos era de casi 2000 (Kovic, Lulic, Brumini, 2008), lo cual permite ilustrar la importancia de la blogosfera médica. Siguiendo las informaciones aportadas por los mismos autores, los tres datos que mejor permiten definir el perfil de estos blogueros son: a) alto nivel de formación (el 71\% había realizado estudios de maestría o doctorado), b) conocimiento elevado del mundo de las revistas científicas y c) realización de una actividad profesional relacionada con el mundo sanitario. Los blogs se han convertido en una fuente de información médica para numerosos pacientes, quienes conceden cada vez una mayor credibilidad a este tipo de soporte informativo. Así, según los datos aportados por Pew \& American Life Project y California Healthcare Foundation (2009), el 59\% de las personas que buscan información médica ha realizado una de las siguientes acciones: a) lectura de comentarios en los servicios de noticias de las páginas web o de los blogs; b) consulta de los rankings de médicos y hospitales; c) suscripción a servicios de noticias de actualidad sobre temas sanitarios; y d) escucha de podcasts.

Los blogs pueden enriquecer la comunicación institucional del hospital. Por un lado, estas herramientas ayudan a la organización a implicar más a los usuarios en la construcción colectiva de conocimientos (Boulos, Maramba, Wheeler, 2006), y por otro, pueden ayudar a dichas instituciones a promover la educación del paciente en los temas sanitarios (Adams, 2010). Esta situación ha llevado a varios hospitales a apostar por este soporte comunicativo, lo cual se plasma en la posibilidad ofrecida al paciente de participar en diferentes blogs sanitarios especializados en patologías, los cuales son accesibles desde el sitio web oficial del hospital. Dicha institución se encarga de gestionar los blogs y de facilitar al paciente el contacto con otros que sufren patologías similares así como con los propios profesionales de la salud que trabajan en el centro hospitalario. Esta iniciativa resulta coherente con el protagonismo asumido por el profesional de la salud y el paciente ya que ambos pueden comunicarse de modo libre gracias al hospital, el cual ejerce una la- 
bor de intermediación pero no de intromisión. Esta labor de intermediación tiene como único objetivo garantizar la calidad de la información médica transmitida al paciente y, por ende, facilitar la educación de este último en los temas sanitarios.

Además de los blogs, los hospitales también recurren a las redes sociales para mejorar sus relaciones comunicativas con los diferentes grupos de interés. La mayor parte de los usuarios de internet de menos de 30 años se han convertido en usuarios activos de las redes sociales (Sublet, Spring, Howard, 2011), lo cual ha dado lugar al surgimiento de redes sociales genéricas (Facebook, MySpace, etc.), pero también redes sociales especializadas en diferentes temas, entre ellos, la salud, como es el caso, por ejemplo, de PatientsLikeMe o de Tsalud.com, donde los médicos aconsejan a los pacientes sobre distintos temas y donde también los propios pacientes pueden interactuar entre sí para compartir experiencias sanitarias. Las redes sociales constituyen una oportunidad estratégica para los hospitales que desean instaurar una comunicación institucional más participativa. Esta situación ha llevado a algunos hospitales a crear perfiles institucionales en diferentes redes sociales, los cuales además son promocionados en la propia página web del hospital. Además, gracias a las redes sociales, los hospitales pueden conocer mejor a los pacientes, especialmente las percepciones que estos tienen sobre los temas de salud (Keelan et al. 2010), lo cual puede facilitar la adaptación de la estrategia de comunicación 2.0 del hospital a las expectativas y necesidades del paciente.

Desde el punto de vista del paciente, la comunicación hospitalaria a través de las redes sociales puede aportarle varios beneficios. Por ejemplo, es el caso de las aplicaciones virtuales de la red social Second Life, que ayudan a los ancianos y a los enfermos a combatir la soledad y el aislamiento (Kamel Boulos, Hetherington, Wheeler, 2007), lo cual puede influenciar positivamente en el restablecimiento emocional del paciente. Asimismo, se puede destacar el caso de MySpace, donde el paciente puede integrarse en grupos de autoayuda, como es el caso de MySpace Cure Diabetes Group (McLean, Richards, Wardman, 2007). Además del apoyo emocional, las redes sociales también facilitan al paciente la gestión de algunas de sus datos médicos, como por ejemplo los resultados de las pruebas diagnósticas. Sin embargo, existe un debate sobre si los ciudadanos pueden manipular su propia información clínica (Tang, Lee, 2009), lo cual no sólo supone un reto desde un punto de vista informativo (capacidad para comprender ciertas informaciones) sino también jurídico (confidencialidad y privacidad del paciente). Algunos autores como O'Keeffe y Clarke-Pearson (2011) han identificado la denominada "de- 
presión por Facebook", que se da en jóvenes que pasan mucho tiempo en esta red social y que exhiben en su "muro" síntomas típicos de depresión.

A pesar de las ventajas y desventajas citadas, las redes sociales se han generalizado en el ámbito sanitario y, de hecho, según el informe Bupa Health Pulse, basado en una encuesta realizada a 12000 personas de Australia, Brasil, Reino Unido, China, Francia, Alemania, India, Italia, México, Rusia, España y Estados Unidos, el $18 \%$ de los usuarios ya utiliza internet para acceder a redes sociales como Facebook que les informen sobre temas de salud (McDaid, 2011). Así, los contenidos de salud en Twitter ya representan el 4\% y en Youtube, el 6\%; y, por otro lado, la ciencia, en su conjunto, representa el $10 \%$ de los contenidos de la blogosfera (Pew Research Center's Project for Excellence in Journalism, 2011). A modo de ejemplo, se puede citar el caso del Hospital Italiano de Buenos Aires, que cuenta con un campus virtual en Facebook destinado a la educación sanitaria para profesionales de la salud de diferentes países, y que ya cuenta con 2403 personas que visitan la página habitualmente ${ }^{1}$.

Por último, la tercera iniciativa que un hospital puede incorporar a su estrategia de comunicación 2.0 es el teléfono móvil. Según Reuther (2008), este dispositivo es una herramienta de comunicación que complementa de un modo eficaz a otros soportes comunicativos como por ejemplo el sitio web. Por ello, cada vez resulta más común que los hospitales dispongan en su portal diversas aplicaciones para el teléfono móvil (petición de consulta médica a través del envío de un SMS, etc.), las cuales facilitan el contacto constante del paciente con el profesional de la salud, lo que permite a este último convertirse en protagonista de la comunicación hospitalaria 2.0. Además, el teléfono móvil permite mejorar las relaciones terapéuticas que el profesional de la salud establece con el paciente (Wong, Tan, Drossman, 2010), lo cual influye positivamente en la imagen de marca del hospital. El desarrollo de este tipo de comunicación se ha visto reforzado por el avance tecnológico que ha experimentado el sector de la telefonía móvil y que se ha plasmado especialmente en la comercialización masiva de los smartphones. El intercambio bidireccional de información entre médico y paciente, la asistencia sanitaria a distancia y el desarrollo de las apps en los smartphones son algunos de los factores que permiten comprender el potencial de la telefonía móvil aplicada al ámbito sanitario (Banco Interamericano de Desarrollo, 2009) así como su rol clave en la estrategia de comunicación 2.0 del hospital.

1 Consultar: es-es.facebook.com/campus/virtual. 
Esta realidad ha dado lugar al concepto de mobile salud o $m$-salud, que se define como "la práctica médica realizada con el apoyo de dispositivos móviles, tales como: teléfonos móviles, PDAs y otros dispositivos inalámbricos" (Organización Mundial de la Salud, 2011). Este tipo de dispositivos necesitan ser diseñados de tal modo que ayuden al profesional de la salud a mejorar la comunicación cara a cara y la comunicación no verbal con el paciente (Alsos, Das, Svanaes, 2012). Para mejorar dicha comunicación, una de las aplicaciones más importantes de la $m$-salud son las tablets, las cuales, según González (2012), deben disponer de ciertas características técnicas como por ejemplo la definición mínima para interpretar imágenes clínicas con precisión, la velocidad de su procesador y de su RAM (random acces memory) y la conectividad con las redes locales o inalámbricas. A modo de ejemplo, merece especial atención el caso del Hospital Universitario Austral de Buenos Aires, en el que los profesionales de la salud disponen de tablets para acceder a la base de datos y a las historias clínicas de los pacientes ${ }^{2}$.

\section{Conclusiones}

Una vez analizado el rol de la comunicación institucional 2.0 en el contexto hospitalario, y teniendo en cuenta las numerosas limitaciones de este artículo (como por ejemplo la falta de información sobre las estrategias precisas de los diferentes hospitales, o sobre las percepciones que tienen los pacientes sobre las aplicaciones 2.0 implantadas por los hospitales), podemos afirmar que los criterios principales que debe considerar un hospital a la hora de fijar su estrategia de comunicación institucional 2.0 son cinco: 1) combinar el enfoque comercial con el enfoque pedagógico centrado en la formación del paciente en temas de salud, 2) implicar a los profesionales de la salud en las acciones de comunicación 2.0,3) complementar las acciones de comunicación 2.0 con acciones de comunicación interpersonal que tengan lugar en el hospital de modo presencial, 4) respetar la coherencia entre las acciones de comunicación 2.0 y la política de comunicación institucional del hospital, y 5) respetar la ética en todas sus dimensiones (comunicativa, hospitalaria, etc.) así como proteger el valor de la dignidad del paciente.

\footnotetext{
2 www.hospitalaustral.edu.ar/institucional_infraestructuras.asp
} 


\section{Referencias}

Adams, S. (2010). Blog-based applications and health information: two case studies that illustrate important questions for consumer health informatics (CHI) research. International Journal of Medical Informatics, 79 (6), e89-e96.

Alsos, O., Das, A. \& Svanaes, D. (2012). Mobile health IT: the effect of user interface and form factor on doctor-patient communication. International Journal of Medical Informatics, 81 (1), 12-28.

Argenti, P. A. (2003). Corporate communication. Boston: McGraw-Hill/Irwin.

Asenjo, M. A. (2002). Las claves de la gestión hospitalaria. 2ª ed. Barcelona: Gestión 2000.

Banco Interamericano de Desarrollo (2009). Salud móvil: el potencial de la telefonía celular para llevar la salud a la mayoría. Washington: Banco Interamericano de Desarrollo.

Barret, R. (2003). Libérer l'âme de l'entreprise : bâtir une organisation visionnaire guidée par les valeurs. Bruxelles: De Boeck.

Boulos, M., Maramba, I. \& Wheeler, S. (2006). Wikis, blogs and podcasts: a new generation of webbased tools for virtual collaborative clinical practice and education. BMC Medical Education, 6, 41.

Buckley, P. T. (2007). The complete guide to hospital marketing. Marblehead: HCPro Inc.

Chui, M., Miller, A. \& Roberts, R. G. (2009, Feb.). Six ways to make web 2.0 work. The McKinsey Quarterly, Consultado el 2 de junio de 2012 de: http://www.mckinseyquarterly.com/ Six_ways_to_make_Web_20_work_2294.

Costa, J. (2001). Imagen corporativa en el siglo XXI. Buenos Aires: La Crujía.

Fisher, J., Burstein, F., Lynch, K. \& Lazarenko, K. (2008). Usability + usefulness = trust: an exploratory study of Australian health web sites. Internet Research, 18 (5), 477-498.

Fostier, P. (2005). L'influence de l'internet sur la communication médecin-patient. En: Richard, C. \& Lussier, M. (eds.). La communication professionnelle en santé (p. 693-714). Québec: ERPI.

Giustini, D. (2006). How web 2.0 is changing medicine. British Medical Journal, 333 (7582), p. 1283-1284.

González, T. (2012). El cambio global de la medicina 2.0. En: Medina, P \& González, T. (eds.). La comunicación médica interactiva (p.101-126). Madrid: Fragua.

Guttman, N. (2003). Ethics in health communication interventions. En: Thompson, T., Dorsey, A., Parrott, R. \& Miller, K. (eds). Handbook of health communication (p. 651-679). Mahwah: Lawrence Erlbaum Associates.

Hesse, B., Nelson, D., Kreps, G., Croyle, R., Arora, N., Rimer, B. \& Viswanath, K. (2005). Trust and sources of health information: the impact of the internet and its implications for health care providers: findings from the first health information national trends survey. Archives of Internal Medicine, 165 (22), 2618-2624.

Huntington, P., Nicholas, D. \& Williams, P. (2003). Characterising and profiling health web user and site types: going beyond hits. Aslib Proceedings, 55 (5/6), 277-289.

Kamel Boulos, M., Hetherington, L. \& Wheeler, S. (2007). Second life: an overview of the potential of 3-D virtual worlds in medical and health education. Health Information and Libraries Journal, 24, 233-245.

Keelan, J., Pavr, V., Balakrishnan, R. \& Wilson, K. (2010). An analysis of the human papilloma virus vaccine debate on MySpace blogs. Journal of Computer-Mediated Communication, 28 (6), 1535-1540.

Kivits, J. (2009). Everyday health and the Internet: a mediated health perspective on health information seeking. Sociology of Health \& Illness, 31 (5), 673-687. 
Kovic, I., Lulic, I. \& Brumini, G. (2008). Examining the medical blogosphere: an online survey of medical bloggers. Journal of Medical Internet Research, 10 (3), e28.

Lemire, M. (2009). Internet et responsabilisation: perspective de l'usager au quotidien. Santé Publique, 21 (2), 13-25.

McDaid, D. (2011). Online health: untangling the web. Consultado el 2 de junio de 2012 de:

http://www.bupa.com/media/44806/online_20health_20_20untangling_20the_20web.pdf.

McKee, M. \& Healy, J. (eds.) (2002). The role and function of hospitals. En su: Hospitals in a changing Europe (p. 59-80). Buckingham: Open University Press.

McLean, R., Richards, B. \& Wardman, J. (2007). The effect of web 2.0 on the future of medical practice and education: Darwikinian evolution or folksonomic revolution? The Medical Journal of Australia, 187 (3), 174-177.

Mintzberg, H. (2004). Managers not MBAs: a hard look at the soft practice of managing and gestion development. London: Prentice Hall, Financial Times.

Moyá Garrido, M. N. (2009). Internet, salud y ciudadanía. Sevilla: Agencia de Evaluación de Tecnologías Sanitarias.

Mucchielli, A. (2005). Information et communication interne: étude des communications: pour de nouveaux audits. 2e. ed.). Paris: Armand Colin.

Nieto, A. (2008, 10 de marzo). Comunicación institucional: bases para la evaluación. Ideas tomadas en el curso de doctorado impartido por don Alfonso Nieto en la Facultad de Comunicación de la Universidad de Navarra.

O'Keeffe, G. S. \& Clarke-Pearson, K. (2011). The impact of social media on children, adolescent and famílies. Pediatrics, 127, 800-804.

Organización Mundial de la Salud (2011). Global observatory for ehealth. Consultado el 2 de junio de 2012 de: http://www.who.int/goe/mobile_health/en/

Parsons, P. J. (2001). Beyond persuasion: the healthcare manager's guide to strategic communication. Chicago: Health Administration Press.

Pew \& American Life Project, California Healthcare Foundation (2009). The social life of health information. Washington DC: Pew \& American Life Project, California HealthCare Foundation.

Pew Research Center's Project for Excellence in Journalism (2011). New media, old media. Consultado el 2 de junio de 2012 de: http://www.journalism.org/analysis_report/blogosphere.

Rafalski, E. \& Mullner, R. (2003). Ensuring HIPAA compliance using data warehouses for healthcare marketing. Journal of Consumer Marketing, 20 (7), 629-633.

Real Academia Española (2001). Diccionario de la lengua española. 22a. ed. Madrid: Espasa-Calpe.

Reid, G. (2008). Building an accreditation scheme for health and social care information. Health Info Internet, 61 (1), 3-5.

Reuther, P. (2008). Mobile search work for the mobile ecosystem: implications for operators, portals, advertisers, and brands. International Journal of Mobile Marketing, 3 (2), 25-33.

Sierra Caballero, F. (2002). Comunicación, educación y desarrollo: apuntes para una historia de la comunicación educativa. Sevilla: Comunicación Social.

Sublet, V., Spring, C. \& Howard, J. (2011). Does social media improve communication? Evaluating the NIOSH science blog. American Journal of Industrial Medicine, 54 (5), 384-394.

Tang, P. \& Lee, T. (2009). Your doctor's office or the internet? Two paths to personal health records. The New England Journal of Medicine, 360 (13), 1276-1278. 
Van Riel, C. B. M. (1998). Comunicación corporativa. Madrid: Prentice Hall.

Van Riel, C. B. M. \& Fombrun, C. J. (2007). Essentials of corporate communication: implementing practices for effective reputation gestion. Abingdon: Routledge.

Wong, R., Tan, J. \& Drossman, D. (2010). Here's my phone number, don't call me: physician accessibility in the cell phone and e-mail era. Digestive Disease and Sciences, 55 (3), 662-667.

Wright, K. B., Sparks, L. \& O'Hair, D. (2008). Health communication in the 21st century. Malden: Blackwell.

\section{Correspondencia}

\section{Pablo Medina Aguerrebere}

Universitat Internacional de Catalunya

Calle Inmaculada, 22

08017 Barcelona

pmedina@uic.es 